\title{
Free-Standing Si and Ge, and Ge/Si Core-Shell Semiconductor Nanowires
}

\author{
H. Peelaers ${ }^{a, b, *}$, B. Partoens ${ }^{a}$ And F.M. Peeters ${ }^{a}$ \\ ${ }^{a}$ Universiteit Antwerpen, Departement Fysica, Groenenborgerlaan 171, B-2020 Antwerpen, Belgium \\ ${ }^{b}$ Materials Department, University of California, Santa Barbara, CA 93106-5050, USA
}

The properties of free-standing silicon and germanium nanowires oriented along the [110] direction are studied using different first principles methods. We show the corrections due to quasi-particles to the band structures obtained using the local-density approximation. The formation energies of $\mathrm{B}$ and $\mathrm{P}$ doped nanowires are calculated, both in the absence and presence of dangling bond defects and we link these to experimental results. Furthermore, we report on the phonon properties of pure Si and Ge nanowires, as well as Ge/Si core-shell nanowires, and discuss the differences between them.

PACS: $61.46 . \mathrm{Km}, 61.72 . u f, 62.23 . \mathrm{Hj}, 63.22 . \mathrm{Gh}$

\section{Introduction}

Free-standing nanowires have attracted a lot of scientific interest recently, which is due to the promising applications of these wires in devices and the fact that they can now be reproducibly realized experimentally using the vapor-liquid-solid (VLS) method [1]. With these nanowires several nanostructures are already fabricated, such as FETs [2], $p-n$ diodes [3, 4], solar cells [5], and also sensors for chemical and biological substances $[6,7]$. Continued progress towards integrated nanoelectronic circuits will require advances in our ability to better control the electronic properties of these building blocks and to assemble them into increasingly complex structures. Considerable efforts focused on doping Si and Ge nanowires to control their electrical properties. However, doping of nanostructures remains a challenge as a result of both fundamental synthetic issues and statistical fluctuations that are intrinsic to homogeneous doping of small structures. Moreover, charged dopant centers will limit the mobility and the corresponding performance of these semiconductor nanowires. The growth of thin wires has also been reported. For Si, these are mainly oriented along the [110] direction [8], while for Ge both the [111] and [110] directions are reported $[9,10]$. In this work we will focus on the [110] direction.

Motivated by the possibilities these nanowires offer, several theoretical $a b$ initio studies have been performed in order to investigate the wire properties, such as the electronic structure of bare and passivated nanowires $[11,12]$, the properties of doped nanowires [13-17] and

\footnotetext{
* corresponding author; e-mail: Hartwin.Peelaers@ua.ac.be
}

the phonon related properties [18, 19]. A thorough overview of the theoretical studies can be found in the recent review paper by Rurali [20].

In this paper we first investigate the structure of undoped Si and Ge nanowires by using quasiparticle corrected band structures. This is followed by a detailed study of the electronic properties of $\mathrm{B}$ and $\mathrm{P}$ doped nanowires. Then the phonon properties of free standing pure nanowires and of $\mathrm{Ge} / \mathrm{Si}$ core-shell nanowires are reported.

\section{Electronic structure}

Firstly, we study the electronic structure of small diameter undoped Si and Ge nanowires. For this we use first-principles calculations within the density-functional theory (DFT), where the local-density approximation (LDA) is used to describe the exchange-correlation functional. The present results have been obtained with the ABINIT code [21] and the Troullier-Martin [22] pseudopotentials. A kinetic energy cutoff of $20 \mathrm{Ha}$ was used for the undoped and P-doped Si and Ge nanowires (NWs), and of $30 \mathrm{Ha}$ for the B-doped NWs. The Brillouin zone was sampled using the Monkhorst-Pack [23] grids and all structures were relaxed without imposing any symmetry constraints. The structures are considered to be relaxed if the largest force component is smaller than $5 \times 10^{-5} \mathrm{Ha} /$ Bohr. Due to the periodic boundary conditions a layer of vacuum was used in the $x$ and $y$ direction to create an infinite one-dimensional system.

This methodology has however a well-known drawback: it severely underestimates the size of the bandgap. Bulk Ge is even predicted to be metallic. The reason for this underestimation is that LDA does not take 
the many-body interactions between electrons into account. Therefore we use many-body perturbation theory (MBPT) to include these interactions. We calculated the first-order corrections to the DFT-LDA results, using Hedin's GW approximation [24] for the electron self-energy, which is the so-called $\mathrm{G}_{0} \mathrm{~W}_{0}$ approach [25].

When using this for low-dimensional systems special care should be given to the convergence of the vacuum size, the number of $\boldsymbol{k}$-points, and the number of unoccupied bands included in the calculation. Achieving convergence is more difficult, as in the GW approach we are dealing with a charged system (since we add an electron or hole to the system). This causes long range Coulomb interactions between the wire and its neighboring images. This affects the vacuum size needed and the number of $\boldsymbol{k}$-points. Part of this problem can be solved by introducing a cutoff on this Coulomb interaction. Here we used the Ismail-Beigi method [26]. In order to reduce the number of unoccupied bands needed in the calculation, an extrapolar method [27] was used. Even with these methods, calculating a full band structure is very resource intensive, as all $\boldsymbol{k}$-points of the desired band structure should be part of a regular grid. Therefore we used a band interpolation scheme based on maximally-localised Wannier functions [28, 29]. Details of these methods and the convergence are reported elsewhere [30].

Figure 1 shows the band structures for a $1.2 \mathrm{~nm} \mathrm{Ge}$ nanowire, obtained using the method described before. The solid red lines correspond to the $G_{0} W_{0}$ corrected LDA band structure, which is also shown as black dots. The LDA band structure is scissor shifted to obtain the same bandgap (so from $1.57 \mathrm{eV}$ to $2.98 \mathrm{eV}$ ). Both band structures use the valence band maximum as energy reference. It can be observed that this nanowire has a direct bandgap, something that is also correctly predicted within LDA. The other wires also have direct bandgaps. The magnitude of the bandgap in LDA is however underestimated. From this figure it can be seen that the agreement between the (scissor shifted) LDA and $\mathrm{G}_{0} \mathrm{~W}_{0}$ bandstructure is good for $\boldsymbol{k}$-points close to the $\Gamma$-point and for bands close to the highest valence band and lowest conduction band.

\section{3. $B$ and $\mathbf{P}$ doped nanowires}

Being able to selectively control the bandgap and the Fermi level allows to control properties like photoluminescence and conductivity which is of primary importance for the development of new applications. Therefore a detailed understanding of the electronic properties of doped Si and Ge NWs and the localization of the dopants is important. As dopants boron and phosphorus are considered, as these are also experimentally used. Boron will act as an electron acceptor, as it lacks one electron, while phosphorus has an additional electron and will therefore act as an electron donor.

To study the localization of these dopants in NWs, the formation energy is calculated. This formation energy

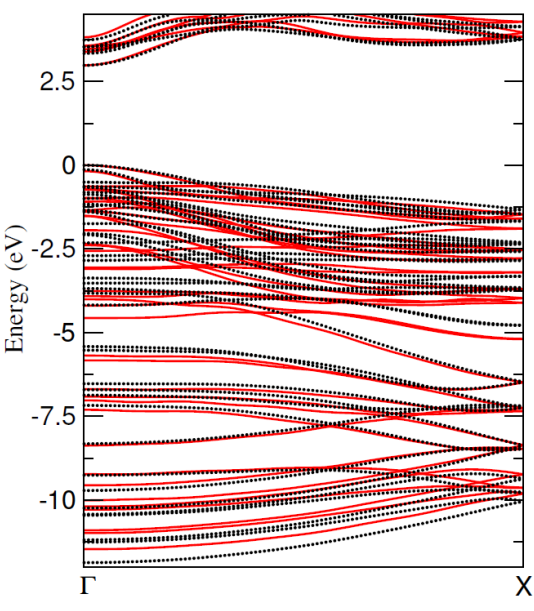

Fig. 1. The $\mathrm{G}_{0} \mathrm{~W}_{0}$ corrected LDA bandstructure of a $1.2 \mathrm{~nm}$ Ge nanowire (solid red). For reference the LDA band structure is also shown (black dots). This bandstructure is scissor shifted to have the $\mathrm{G}_{0} \mathrm{~W}_{0}$ bandgap. Both bandstructures are aligned so that the valence band maximum is at $0 \mathrm{eV}$.

$E_{\mathrm{f}}$ of the impurity $\mathrm{B}$ and/or $\mathrm{P}$ can be defined as the energy needed to insert an atom B and/or P (taken from a reservoir) into the wire after removing one (or more) $\mathrm{Si}$ atoms (to a reservoir, assumed to be bulk $\mathrm{Si}$ )

$$
\begin{gathered}
E_{\mathrm{f}}=E(\text { doped SiNW })-E(\operatorname{SiNW}) \\
+(n+m) \mu_{\mathrm{Si}}-n \mu_{\mathrm{B}}-m \mu_{\mathrm{P}},
\end{gathered}
$$

where $n$ and $m$ are the number of, respectively, $\mathrm{B}$ and $\mathrm{P}$ dopants, $\mu_{\mathrm{Si}}$ the chemical potential of $\mathrm{Si}$ (here set equal to the total energy per atom of bulk $\mathrm{Si}$ ) and $\mu_{\mathrm{B} / \mathrm{P}}$ the chemical potential of the impurity atom. For these chemical potentials we use the total energy per atom in the tetragonal $B_{50}$ crystal for $\mathrm{B}$ and the orthorhombic black phosphorus for $\mathrm{P}$.

In Fig. 2 the formation energy is plotted as function of the dopant position (the different labels correspond to the atom labels shown in the inset) for $\mathrm{P}$ (left) and $\mathrm{B}$ (right) dopants in a $1.6 \mathrm{~nm} \mathrm{Si} \mathrm{NW}$. The energy difference $E_{\mathrm{S}}$ is the segregation energy and is defined as the energy difference between a dopant in the center of the wire and one near the wire edge.

One can clearly see that both $\mathrm{B}$ and $\mathrm{P}$ dopant prefer the edge position, but not directly bonded to a $\mathrm{H}$ atom (position labeled 4 in the right-handside inset). The same result for the Si NW was found in the thinner wire (also depicted in the inset). For Ge NWs however, we find that the B dopant prefers the edge position (position 5), as shown in Fig. 3. The behavior for the $\mathrm{P}$ dopant is similar as in the Si NW, however, the segregation energy (energy difference between the wires with the dopant at the central position and the dopant at the preferred position) is much smaller ( $142 \mathrm{meV}$ in the large SiNW, and only $60 \mathrm{meV}$ in the large GeNW).

The reason why these near edge positions are energetically favorable is a relaxation effect: in the more centrally 


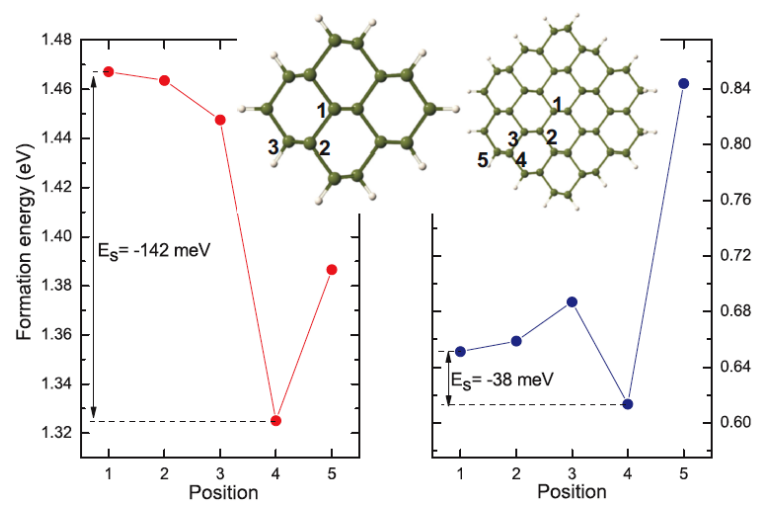

Fig. 2. The formation energy as function of the position of the $\mathrm{P}$ dopant (left) and $\mathrm{B}$ dopant (right) in a $1.6 \mathrm{~nm}$ nanowire. The inset shows a top view of a 1.2 and $1.6 \mathrm{~nm} \mathrm{H}$ passivated wire along the [110] direction.

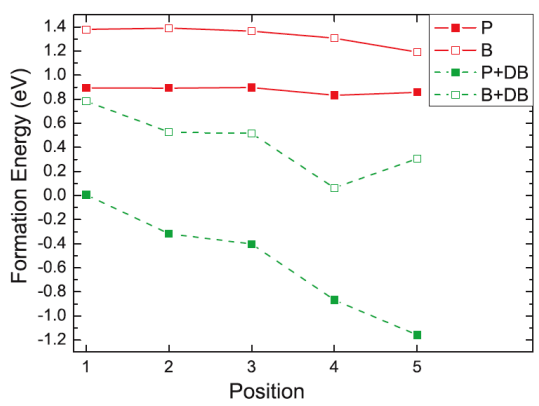

Fig. 3. The formation energy as function of the position of a $P$ (filled squares) and B (open squares) dopant in a $1.6 \mathrm{~nm}$ Ge nanowire. The lines are a guide to the eye. Full (dashed) lines correspond to dopants in the absence (presence) of dangling bonds.

located positions the allowed relaxation is smaller due to the stability of the Si or Ge structure.

Another effect that can be seen is that the general trend for $\mathrm{P}$ dopants is that the formation energy decreases when going towards the edge of the nanowire, while the opposite is true for B dopants (ignoring position 4). Therefore one would expect to have more $\mathrm{P}$ dopants closer to the edge of the wire than in the center of the wire.

Because the diameter of the studied wires is very small, these wires have a large surface to volume ratio, and therefore the number of dopants can be smaller or equal to the number of dangling bonds (DBs) (corresponding to $\mathrm{Si}$ or Ge atoms at the surface which are not saturated with a hydrogen atom). These dangling bonds are chemical very reactive. So they can have an important impact on the electronic properties. Therefore we also studied the effect of such a dangling bond on the preferred positions and on the segregation energies.

The effect of the presence of a DB is illustrated in Fig. 3, which shows the formation energy for a $1.6 \mathrm{~nm}$ Ge NW. A dangling bond lowers the formation energy for both types of dopants, thus making it easier to dope the wire. The preferred position for a $\mathrm{P}$ dopant changes to the position near the dangling bond. The segregation energy to this position is $-1.09 \mathrm{eV}$. This large segregation energy indicates that the dangling bond will attract the P-type dopants. For a B dopant the segregation energy is equal to $476 \mathrm{meV}$. This is only half of the segregation energy for a $\mathrm{P}$ dopant. Thus for the same concentration of dopants, the fraction of $\mathrm{P}$ atoms that will be trapped is larger than the fraction of $\mathrm{B}$ atoms. So the concentration of free carriers will be larger for B doping than for $\mathrm{P}$ doping. This is in agreement with experimental conductance measurements which show a higher resistivity for $\mathrm{P}$ doped Si wires than for B doped Si wires, for the same level of concentration [31, 32] and also agrees with the theoretical results of Ref. [14].

\section{Phonon properties}

Knowledge of the behavior of phonons in a material can be important for several applications, as the thermal properties of semiconducting systems, such as the thermal conductance, are largely determined by phonons. Another example is the low-field mobility, which for not too low temperatures is limited due to scattering on acoustic phonons. Phonon spectra are also useful to determine the stability of structures [33].

Here we calculated the phonon band structure using density functional perturbation theory (DFPT) [34], as this approach does not require the use of large supercells.

In Fig. 4 the phonon spectrum for a $1.2 \mathrm{~nm} \mathrm{Si} \mathrm{NW}$ is shown (top). Frequencies larger than $1000 \mathrm{~cm}^{-1}$, corresponding to $\mathrm{Si}-\mathrm{H}$ stretching modes are not shown, but these can be seen in the phonon density-of-states of Fig. 5a. The spectrum is real and positive, which indicates that the considered nanowire is structurally stable. From our calculations it follows that the same is true for Ge NWs and B or P doped nanowires (if the diameter is at least $1.2 \mathrm{~nm}$ ) [18, 19]. The lower part of that figure (Fig. 4b) zooms in on the acoustic branches of the undoped $1.2 \mathrm{~nm}$ wire near the $\Gamma$ point. Four zero-frequency modes can be observed, three acoustic modes that are related to the translational symmetry and one extra acoustic mode (not present in bulk) that is related to the rotational invariance of the wire around the wire axis. Two of these modes are linear in $q_{z}$, and correspond to displacements along the wire axis, i.e., a translational mode along the wire axis and the other is a rotational mode, i.e., torsion of the wire. The two other modes are proportional to $q_{z}^{2}$. These softer modes are eigenmodes with displacements orthogonal to the wire axis and correspond to a bending of the wire. The eigenvectors of the rotational mode can be seen in the inset (a) of Fig. 4b, which shows the calculated eigenvectors of this mode for a larger $(1.6 \mathrm{~nm}) \mathrm{Si}$ wire. Inset (b) of Fig. $4 \mathrm{~b}$ shows the longitudinal translational mode (also linear), and insets (c) and (d) show the quadratic orthogonal modes. The shown eigenvectors are normalized with respect to the 

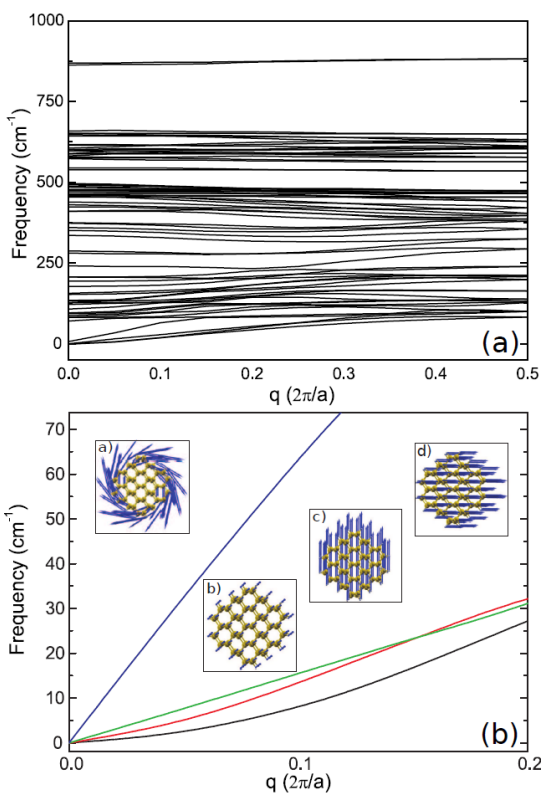

Fig. 4. (a) The phonon spectrum of a $1.2 \mathrm{~nm} \mathrm{Si}$ nanowire. Frequencies above $1000 \mathrm{~cm}^{-1}$ are not shown. (b) A zoom of the spectrum for the region of low frequencies close to the $\Gamma$-point. The insets show the eigenvectors of the phonon modes for the larger $1.6 \mathrm{~nm} \mathrm{Si}$ nanowire for (a) the rotational mode, (b) the longitudinal mode, and (c) and (d) show the transversal modes.

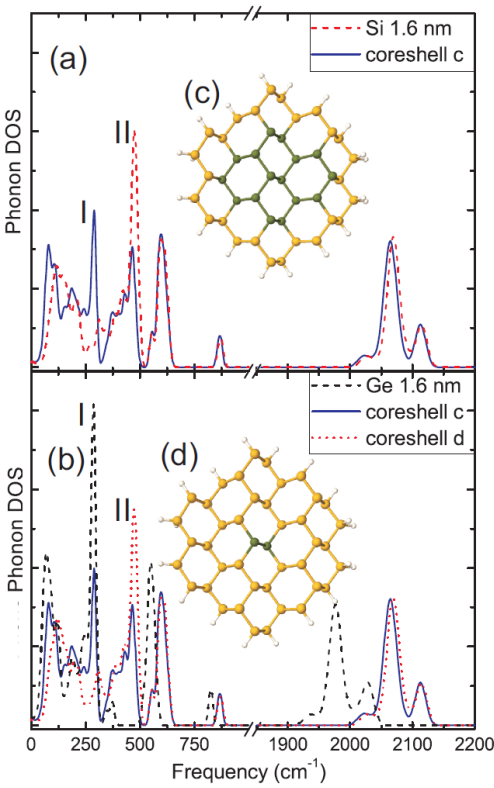

Fig. 5. The phonon density of state of a $1.6 \mathrm{~nm} \mathrm{Si}$ and Ge nanowire compared to two different $\mathrm{Ge} / \mathrm{Si}$ core-shell structures, which are shown in the insets. The labels core-shell $c$ and core-shell $d$ refer to the core-shell structure (c) and (d). (a) shows the comparison between the pure Si NW (dashed red curve) and coreshell c (full blue curve), while (b) shows the comparison between a pure Ge nanowire (dashed black curve) and both types of core-shell structures (full blue and dotted red curves). atomic mass and multiplied by a factor for displaying purposes.

\section{Core-shell nanowires}

Other interesting one-dimensional systems are the so-called coaxial heterostructures or core-shell nanowires. Coaxial $\mathrm{Si} / \mathrm{Ge}$ heterostructures of such wires can readily be made [35-39]. Changing the sizes of the core and shell regions allows one to engineer the bandgaps, as was shown experimentally [39] and theoretically [40, 41].

Here we focus on the phonon properties of $\mathrm{Ge} / \mathrm{Si}$ core-shell nanowires. For this we will look at the $1.6 \mathrm{~nm}$ nanowire, so that two different types of core-shell structures can be made ( $\mathrm{Ge}$ is always in the core region). Both structures are shown as insets (c) and (d) in Fig. 5. All calculated phonon frequencies were real and positive, so that we can conclude that all considered core-shell nanowires are stable. To have a better understanding on how the structure influences the phonon frequencies, we calculated the phonon density-of-states (DOS) for the $1.6 \mathrm{~nm} \mathrm{Si}, \mathrm{Ge}$ and the two different Ge/Si core-shell structures.

These different DOS are shown in Fig. 5. For reference the phonon DOS of pure Si and Ge NWs are also shown by the dashed curves. Two distinct features of pure Si and Ge NWS are labeled I and II, respectively. From the highest frequency states (around $2000 \mathrm{~cm}^{-1}$ ), which correspond to hydrogen stretching modes, the type of material of the outer shell can be deduced by comparison with the pure spectra. In this case this is clearly a Si shell. The same conclusion can be obtained from the peaks around 600 and $800 \mathrm{~cm}^{-1}$, as these correspond to $\mathrm{Si}-\mathrm{H}$ bending modes. From the occurence in the spectrum of the peak labeled I (a typical Ge peak) and the reduced magnitude of the peak labeled II (a typical Si peak) one can deduce that it is not a pure Si nanowire, but that it also contains Ge.

\section{Conclusions}

In conclusion, we showed that $\mathrm{Si}$ or Ge nanowires oriented along the [110] direction have direct bandgaps and that this is correctly predicted by LDA. This was shown using quasi-particle corrections within the $\mathrm{G}_{0} \mathrm{~W}_{0}$ method. It was shown that the preferential position for $B$ or $\mathrm{P}$ dopants is the near edge position. In the presence of a dangling bond the segregation energy increases and this increase is larger for $\mathrm{P}$ dopants compared to $\mathrm{B}$ dopants, which has experimental consequences. By calculating the phonon spectra we showed that all wires are stable and we explained the low frequency spectrum consisting of two linear and two quadratic modes. When a shell of a different material is added, these phonon spectra change. The nature of this change was explained and it was indicated how this can be used to identify the composition of a particular nanowire. 


\section{Acknowledgments}

This work was supported by the Flemish Science Foundation (FWO-Vl), the Belgian Science Policy (IAP), the NSF MRSEC Program under award No. DMR05-20415, and the Belgian American Educational Foundation.

\section{References}

[1] V. Schmidt, J.V. Wittemann, S. Senz, U. Gösele, Adv. Mater. 21, 2681 (2009).

[2] C. Thelander, P. Agarwal, S. Brongersma, J. Eymery, L. Feiner, A. Forchel, M. Scheffler, W. Riess, B. Ohlsson, U. Gösele, L. Samuelson, Mater. Today 9, 28 (2006).

[3] X. Duan, Y. Huang, Y. Cui, J. Wang, C.M. Lieber, Nature (London) 409, 66 (2001).

[4] Y. Cui, C.M. Lieber, Science 291, 851 (2001).

[5] B. Tian, T.J. Kempa, C.M. Lieber, Chem. Soc. Rev. 38, 16 (2009).

[6] Y. Cui, Q. Wei, H. Park, C.M. Lieber, Science 293 , 1289 (2001).

[7] F. Patolsky, G. Zheng, O. Hayden, M. Lakadamyali, X. Zhuang, C.M. Lieber, Proc. Natl. Acad. Sci. USA 101, 14017 (2004).

[8] Y. Wu, Y. Cui, L. Huynh, C.J. Barrelet, D.C. Bell, C.M. Lieber, Nano Lett. 4, 433 (2004).

[9] H. Jagannathan, M. Deal, Y. Nishi, J. Woodruff, C. Chidsey, P.C. McIntyre, J. Appl. Phys. 100 , 024318 (2006).

[10] D. Wang, H. Dai, Angew. Chem. Int. Ed. 41, 4783 (2002).

[11] M.-F. Ng, L. Zhou, S.-W. Yang, L.Y. Sim, V.B.C. Tan, P. Wu, Phys. Rev. B 76, 155435 (2007).

[12] R. Kagimura, R.W. Nunes, H. Chacham, Phys. Rev. Lett. 95, 115502 (2005).

[13] H. Peelaers, B. Partoens, F.M. Peeters, Nano Lett. 6, 2781 (2006)

[14] M.V. Fernandez-Serra, C. Adessi, X. Blase, Phys Rev. Lett. 96, 166805 (2006).

[15] H. Peelaers, B. Partoens, F.M. Peeters, Appl. Phys. Lett. 90, 263103 (2007).

[16] C.R. Leao, A. Fazzio, A.J.R. da Silva, Nano Lett. 8, 1866 (2008).

[17] R. Rurali, M. Palummo, X. Cartoixa, Phys. Rev. B 81, 235304 (2010).

[18] H. Peelaers, B. Partoens, F.M. Peeters, Nano Lett. 9, 107 (2009).

[19] H. Peelaers, B. Partoens, F.M. Peeters, Appl. Phys. Lett. 95, 122110 (2009).

[20] R. Rurali, Rev. Mod. Phys. 82, 427 (2010).
[21] X. Gonze, B. Amadon, P.-M. Anglade, J.-M. Beuken, F. Bottin, P. Boulanger, F. Bruneval, D. Caliste, R. Caracas, M. Ct, T. Deutsch, L. Genovese, P. Ghosez, M. Giantomassi, S. Goedecker, D. Hamann, P. Hermet, F. Jollet, G. Jomard, S. Leroux, M. Mancini, S. Mazevet, M. Oliveira, G. Onida, Y. Pouillon, T. Rangel, G.-M. Rignanese, D. Sangalli, R. Shaltaf, M. Torrent, M. Verstraete, G. Zerah, J. Zwanziger, Comput. Phys. Commun. 180, 2582 (2009).

[22] N. Troullier, J.L. Martins, Phys. Rev. B 43, 1993 (1991).

[23] H.J. Monkhorst, J.D. Pack, Phys. Rev. B 13, 5188 (1976).

[24] L. Hedin, Phys. Rev. 139, A796 (1965).

[25] W.G. Aulbur, L. Jönsson, J.W. Wilkins, Solid State Phys. 54, 1 (1999).

[26] S. Ismail-Beigi, Phys. Rev. B 73, 233103 (2006).

[27] F. Bruneval, X. Gonze, Phys. Rev. B 78, 085125 (2008).

[28] N. Marzari, D. Vanderbilt, Phys. Rev. B 56, 12847 (1997).

[29] I. Souza, N. Marzari, D. Vanderbilt, Phys. Rev. B 65 , 035109 (2001).

[30] H. Peelaers, B. Partoens, M. Giantomassi, T. Rangel, E. Goossens, G.-M. Rignanese, X. Gonze, F.M. Peeters, Phys. Rev. B 83, 045306 (2011).

[31] Y. Cui, X. Duan, J. Hu, C.M. Lieber, J. Phys. Chem. B 104, 5213 (2000).

[32] J.-Y. Yu, S.-W. Chung, J.R. Heath, J. Phys. Chem. B 104, 11864 (2000)

[33] M.J. Verstraete, X. Gonze, Phys. Rev. B 74, 153408 (2006).

[34] S. Baroni, S. de Gironcoli, A. Dal Corso, P. Giannozzi, Rev. Mod. Phys. 73, 515 (2001).

[35] L.J. Lauhon, M.S. Gudiksen, D. Wang, C.M. Lieber, Nature (London) 420, 57 (2002).

[36] Y. Hu, J. Xiang, G. Liang, H. Yan, C.M. Lieber, Nano Lett. 8, 925 (2008).

[37] W. Lu, J. Xiang, B.P. Timko, Y. Wu, C.M. Lieber, Proc. Natl. Acad. Sci. USA 102, 10046 (2005).

[38] J. Xiang, W. Lu, Y. Hu, Y. Wu, H. Yan, C.M. Lieber, Nature (London) 441, 489 (2006).

[39] J.-E. Yang, C.-B. Jin, C.-J. Kim, M.-H. Jo, Nano Lett. 6, 2679 (2006).

[40] R.N. Musin, X.-Q. Wang, Phys. Rev. B 74, 165308 (2006).

[41] H. Peelaers, B. Partoens, F.M. Peeters, Phys. Rev. B 82, 113411 (2010). 